Phase III randomised trial

\title{
The UK HeartSpare Study: Randomised evaluation of voluntary deep-inspiratory breath-hold in women undergoing breast radiotherapy
}

\author{
Frederick R. Bartlett ${ }^{\mathrm{a}, *}$, Ruth M. Colgan ${ }^{\mathrm{b}}$, Karen Carr ${ }^{\mathrm{a}}$, Ellen M. Donovan ${ }^{\mathrm{b}}$, Helen A. McNair ${ }^{\mathrm{a}}$, \\ Imogen Locke ${ }^{a}$, Philip M. Evans ${ }^{\mathrm{b}, \mathrm{c}}$, Joanne S. Haviland ${ }^{\mathrm{d}}$, John R. Yarnold ${ }^{\mathrm{a}, \mathrm{e}}$, Anna M. Kirby ${ }^{\mathrm{a}}$
}

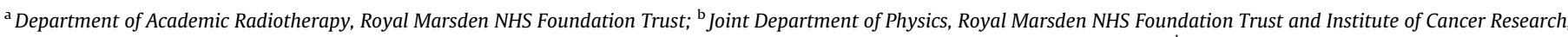


and ${ }^{\mathrm{e}}$ Division of Radiotherapy and Imaging, Institute of Cancer Research, Sutton, UK

\section{A R T I C L E I N F O}

\section{Article history:}

Received 18 December 2012

Received in revised form 24 April 2013

Accepted 26 April 2013

Available online 29 May 2013

\section{Keywords:}

Radiotherapy

Breast cancer

Cardiac dose

Activated breathing control

Voluntary breath-hold

\begin{abstract}
A B S T R A C T
Purpose: To determine whether voluntary deep-inspiratory breath-hold (v_DIBH) and deep-inspiratory breath-hold with the active breathing coordinator ${ }^{\mathrm{TM}}$ (ABC_DIBH) in patients undergoing left breast radiotherapy are comparable in terms of normal-tissue sparing, positional reproducibility and feasibility of delivery.

Methods: Following surgery for early breast cancer, patients underwent planning-CT scans in v_DIBH and ABC_DIBH. Patients were randomised to receive one technique for fractions $1-7$ and the second technique for fractions 8-15 (40 Gy/15 fractions total). Daily electronic portal imaging (EPI) was performed and matched to digitally-reconstructed radiographs. Cone-beam CT (CBCT) images were acquired for $6 / 15$ fractions and matched to planning-CT data. Population systematic $(\Sigma)$ and random errors $(\sigma)$ were estimated. Heart, left-anterior-descending coronary artery, and lung doses were calculated. Patient comfort, radiographer satisfaction and scanning/treatment times were recorded. Within-patient comparisons between the two techniques used the paired $t$-test or Wilcoxon signed-rank test.

Results: Twenty-three patients were recruited. All completed treatment with both techniques. EPIderived $\Sigma$ were $\leqslant 1.8 \mathrm{~mm}$ (v_DIBH) and $\leqslant 2.0 \mathrm{~mm}$ (ABC_DIBH) and $\sigma \leqslant 2.5 \mathrm{~mm}$ (v_DIBH) and $\leqslant 2.2 \mathrm{~mm}$ (ABC_DIBH) (all $p$ non-significant). CBCT-derived $\Sigma$ were $\leqslant 3.9 \mathrm{~mm}$ (v_DIBH) and $\leqslant 4.9 \mathrm{~mm}$ (ABC_DIBH) and $\sigma \leqslant 4.1 \mathrm{~mm}$ (v_DIBH) and $\leqslant 3.8 \mathrm{~mm}$ (ABC_DIBH). There was no significant difference between techniques in terms of normal-tissue doses (all $p$ non-significant). Patients and radiographers preferred v_DIBH ( $p=0.007, p=0.03$, respectively). Scanning/treatment setup times were shorter for $\mathrm{v}_{-}$DIBH $(p=0.02, p=0.04$, respectively).

Conclusions: v_DIBH and ABC_DIBH are comparable in terms of positional reproducibility and normal tissue sparing. v_DIBH is preferred by patients and radiographers, takes less time to deliver, and is cheaper than ABC_DIBH.
\end{abstract}

(c) 2013 Elsevier Ireland Ltd. All rights reserved. Radiotherapy and Oncology 108 (2013) 242-247
Breast cancer is the most common cancer in women, with a current estimated worldwide incidence of 1.38 million [1]. With improved treatments, the number of breast cancer $(\mathrm{BC})$ survivors is increasing, and in the UK alone is expected to treble to 1.7 million by 2040 [2]. This surge in survivor numbers makes the late effects of BC treatment, including those related to radiotherapy, of increasing concern to patients and healthcare providers alike. Breast radiotherapy reduces the risk of recurrence and improves survival after surgical excision of early BC [3]. However, the benefits derived from breast radiotherapy are compromised by an increase in non-BC deaths, the majority of which are cardiovascular in origin [4]. Although it is not yet clear which cardiac structure(s)

* Corresponding author. Address: Department of Academic Radiotherapy, Royal Marsden NHS Foundation Trust, Downs Road, Sutton SM2 5PT, UK.

E-mail address: frederick.bartlett@rmh.nhs.uk (F.R. Bartlett). is most important in the pathogenesis of radiation related heart disease (RRHD), the anatomical position of the left anterior descending artery (LAD) makes it particularly susceptible to high doses of radiation. There is now mounting evidence that irradiation of the LAD is a key factor in the development of RRHD [5-7]. Irradiation of other tissues, including lung and chest wall, also contributes to late morbidity and mortality from breast radiotherapy [4,8-10].

The current priority in breast radiotherapy is to develop and validate techniques which enable clinicians to maintain coverage of target tissues (breast/chest wall) whilst reducing radiation to adjacent organs-at-risk (OAR), especially the heart and LAD. Breath-holding techniques, in which the heart is pushed down and away from the radiotherapy field, are a solution to this problem. Compared to free-breathing radiotherapy, deep-inspiratory breath-hold with the active breathing coordinator ${ }^{\mathrm{TM}}$ (ABC_DIBH) 
(Elekta, Crawley, UK) significantly reduces the volume of heart irradiated [11-13], whilst voluntary deep-inspiratory breath-hold (v_DIBH) using the Varian RPM system has been shown to significantly reduce median heart and LAD volumes receiving $>50 \%$ of the prescription dose [14-16]. Such dosimetric savings are projected to equate to a 10 -fold reduction in cardiac deaths [16]. Nonetheless, despite convincing evidence that DIBH reduces the dose delivered to cardiac tissues, it was used in only $19 \%$ of EORTC centres 3 years ago [17] and just 4\% of UK centres in 2012 [Royal College of Radiologists audit]. ABC_DIBH has been shown to be reproducible [18] but is expensive to implement due to costs of equipment including daily disposable mouthpieces. v_DIBH, in particular the technique described in this study, would be a low-cost alternative but data on its reproducibility are lacking. This randomised crossover study was designed to answer whether or not the treatment of breast radiotherapy patients in v_DIBH is at least as good as ABC_DIBH in terms of positional reproducibility, normal tissue sparing and feasibility of delivery.

\section{Methods and materials}

This study was approved by the Royal Marsden Committee for Clinical Research and the Research Ethics Committee (ISRCTN 53485935). All women underwent left breast conserving surgery or mastectomy for early stage invasive ductal or lobular carcinoma (pT1-3b N0-1 M0) and were recommended adjuvant radiotherapy to the whole breast or chest wall without nodal irradiation $(+/-$ tumour bed boost). Women with left breast cancer requiring radiotherapy to the breast or chest wall alone were approached. All patients were treated at the Royal Marsden Hospital, Sutton, UK. Randomisation was arranged via telephone at the Clinical Trials and Statistics Unit at the Institute of Cancer Research (ICR-CTSU), Sutton, UK, where patient details were recorded and order of breath-holding techniques was allocated. Randomisation was not blinded. Computer-generated random permuted blocks of size 4 were used for allocation, with no stratification.

\section{v_DIBH technique}

The v_DIBH technique used in this study required no additional equipment. Patients were asked to breathe in and out twice before being asked to take a deep breath and hold. Breath-hold consistency was checked at CT and treatment by the distance moved by the laser from the anterior and lateral tattoos in breath-hold. All breath-hold techniques require a method of monitoring intrafraction breath-hold reproducibility. In this study the borders of the light field were marked with ink with the patient in breathhold. Treatment room cameras were zoomed so that the pen marks and light field were visible on the control room monitors. Visualising these enabled radiographers to check breath-hold consistency prior to and during treatment. A more detailed account of the $\mathrm{v} \_$DIBH technique is given in the supplementary material.

\section{Patient positioning and image acquisition}

Training was given to patients for both techniques immediately prior to scanning. ABC_DIBH training followed the method described by Remouchamps et al. [18] and for v_DIBH is described in the supplementary material. Both CT scans were performed in one CT-planning session and patients remained on the CT couch between scans. All patients were scanned on a supine breast board, with arms extended above the head in supports (Med-Tec, Iowa, USA). Markers were placed bilaterally $1-2 \mathrm{~cm}$ posterior to the mid-axillary line and aligned axially with a midline marker using lateral lasers. CT data (Philips Medical Systems, UK) were acquired without contrast in both ABC_DIBH and v_DIBH using $3 \mathrm{~mm}$ slices from $\mathrm{C} 6$ to below the diaphragm. The time taken to complete each planning session was recorded, from the time the patients mounted the CT couch to the time at which they dismounted the couch. After both scans were completed patients were asked to complete validated comfort and acceptability questionnaires and radiographers were asked to complete radiographer satisfaction questionnaires [19].

\section{Target and organ-at-risk delineation}

Target and OARs were delineated on both CT scans. The whole breast clinical target volume (WCBTV) encompassed the breast tissue visualised on CT (limited by pectoral fascia and $5 \mathrm{~mm}$ from skin). The tumour bed was defined using tumour bed clips (inserted at surgery), and included any associated seroma or distortion of breast architecture. A uniform margin of $15 \mathrm{~mm}$ was added (limited by WBCTV) to form the partial breast CTV (PBCTV). For mastectomy patients, the chest wall CTV (CWCTV) was defined using anatomical landmarks (inferior border of clavicle (superior), $1 \mathrm{~cm}$ below inframammary fold (inferior), midline (medial) and anterior border of serratus anterior (lateral)), taking into account the position of the contralateral breast tissue and limited by pectoral fascia and $5 \mathrm{~mm}$ from skin. The heart was outlined in accordance with the UK National Cancer Research Institute Intensity Modulated and Partial Organ RadioTherapy (IMPORT) study criteria [20]. The LAD was outlined according to the method described by Taylor et al. [21,22]. The lungs were outlined by adapting a preinstalled lung template and edited to exclude major airways.

\section{Radiotherapy planning}

Tangential fields were applied to encompass WBCTV/CWCTV. The depth of lung tissue included in tangential fields was $\leqslant 2.5 \mathrm{~cm}$. Philips Pinnacle 9.2 (Philips Medical Systems, Palo Alto, USA $)$ and the collapsed-cone algorithm $(0.25 \times 0.25 \times 0.25 \mathrm{~cm}$ resolution) were used to produce plans such that $\geqslant 90 \%$ of the WBCTV/CWCTV and $\geqslant 95 \%$ of the PBCTV were covered by the $95 \%$ isodose [23]. Where required, MLC leaves were used to shield cardiac tissue whilst maintaining target tissue constraints. Segments (field-in-field technique) were used, where necessary, to improve dose homogeneity and all plans fulfilled ICRU 62 criteria (dose variation $\leqslant+7 \%$ and $-5 \%$, hotspots $\leqslant 107 \%$ ) [24]. Patients were prescribed $40 \mathrm{~Gy}$ in 15 fractions over 3 weeks using 6 or 10 MV photons.

Tabular DVH data (dose (Gy) per voxel) were used to derive the $\mathrm{NTD}_{\text {mean }}$ (a biologically weighted mean of total dose to tissue normalised to $2 \mathrm{~Gy}$ fractions using a standard linear quadratic model [25], $\alpha / \beta=3 \mathrm{~Gy}$ ) for heart, LAD, ipsilateral and whole lungs. In addition, the maximum dose received by the $\mathrm{LAD}\left(\mathrm{LAD}_{\max }\right)$ was estimated.

\section{Radiotherapy delivery}

Patients were randomised to receive one treatment technique for fractions 1-7 and the second technique for fractions $8-15$. Patient setup is described in the supplementary material. Real-time electronic portal images (EPI) were acquired daily and matched on-line to DRRs on fractions 1-3 and 8-10 using iView software (Elekta, Crawley, UK). Shifts were applied if errors were $>5 \mathrm{~mm}$ in the $(u, v)$-plane on at least two consecutive days. For study purposes setup errors were measured off-line for every fraction. The left posterior oblique beam (LPO) was treated first and the right anterior oblique beam (RAO) treated second.

On-board $\mathrm{kV}-\mathrm{CT}$ (CBCT) images of the chest were acquired immediately after setup on fractions $1,4,7,8,11$ and 14 using the Elekta Synergy X-ray Volume Imaging System (Elekta, Crawley, 
UK). CBCT procedures have been described previously [26]. CBCT data were acquired for study purposes only and were not used to make isocentre shifts. For technical reasons it was not possible to interrupt CBCTs, meaning that patients could only be in breathhold for about two-thirds of the time taken to complete the CBCT. The CBCT volume was manually registered to the reference planning-CT, and both chest wall and clip-based matches were performed. For chest wall matches, a best-fit match was made for the area of chest wall directly posterior to the target tissue. The correction reference point was set to the isocentre.

On average, four to six breath-holds were required per treatment, with two extra for CBCT imaging.

Times at which patients mounted the couch, the radiotherapy beam was switched on and off and at which patients dismounted the couch, were recorded for every fraction. Patients and radiographers were asked to complete questionnaires on fractions $1,7,8$ and 15.

\section{Statistical methods}

A sample size of 23 patients was estimated to provide $95 \%$ power in order to rule out an excess of $2 \mathrm{~mm}$ in mean displacement (primary outcome) for v_DIBH versus ABC_DIBH, assuming a significance level of 0.05 (1-sided as testing for non-inferiority). EPI displacements were analysed for each beam in the $(u, v)$-plane for every patient $(v$-direction parallel to craniocaudal axis and $u$-direction perpendicular to this) [27]. Mean displacements and standard deviations were compared for ABC_DIBH and v_DIBH treatments for each patient. Using the method described by van Herk [28], population mean displacement (MD), systematic $(\Sigma)$ and random $(\sigma)$ errors were estimated. CBCT registration results were analysed in 3-dimensions for each patient and for both chest wall and clipbased matches; population MD, $\Sigma$ and $\sigma$ were calculated. Paired $t$ tests were used to compare the parameters listed above (population MD, $\Sigma$ and $\sigma$ ), normal tissue doses and timing data (CT and treatment session times) between ABC_DIBH and v_DIBH, using each patient as their own control. Data normality was confirmed using Q-Q plots and Kolmogorov-Smirnov tests (all $p$ non-significant). Patient comfort and acceptability questionnaires were summarised as patient comfort scores ranging from 0 (least comfortable) to 9 (most comfortable). Radiographer satisfaction questionnaires were summarised as radiographer satisfaction scores ranging from 0 (most satisfactory) to 9 (least satisfactory). Scores were calculated for each technique and at each timepoint (CT, first and last fractions). In order to eliminate any time effect, the difference in questionnaire scores between the two techniques at each timepoint was calculated for each patient. Single sample Wilcoxon signed-rank tests were then used to test whether the median of the differences was significantly different from zero.

Statistical analyses were performed using SPSS Statistics Version 19 (IBM, Portsmouth, UK).

\section{Results}

Twenty-three patients were randomised between February and August 2012. The study ended when the final patient completed their radiotherapy course, and there was no long-term trial-related follow-up. All patients completed treatment with both techniques. The median age of patients recruited was 61 years (range 36-82). Nineteen patients (83\%) underwent BCS and four (17\%) underwent mastectomy. Table S1 (supplementary material) shows mean target and OAR volumes for both techniques. Mean WBCTV was similar for both techniques: $677 \mathrm{~cm}^{3}$ (ABC_DIBH) vs $676 \mathrm{~cm}^{3}$ (v_DIBH), as was mean target tissue coverage. There was no significant difference in lung volumes between the two techniques. However, heart volumes were significantly smaller with v_DIBH: $577 \mathrm{~cm}^{3}$ vs $544 \mathrm{~cm}^{3}(p<0.001)$.

Table 1 shows population MD, $\Sigma$ and $\sigma$ estimated using EPI. There was no significant difference between the two techniques in MD, $\Sigma$ or $\sigma$.

Table 2 shows population MD, $\Sigma$ and $\sigma$ for CBCT chest wall and clip-based matches. Twenty-two patients underwent CBCTs and clip-based matches were possible in 18 (4 patients underwent mastectomy). There was no significant difference between the two techniques in $\Sigma$ or $\sigma$ for either chest wall or clip-based matches. MD was less with $\mathrm{v}_{-} \mathrm{DIBH}$ in the anterior-posterior (AP) direction. Errors were generally greater for chest wall matches than for clip-based matches.

There was no significant difference between mean normal tissue doses (Gy) for ABC_DIBH and v_DIBH (standard deviation in brackets): heart $\mathrm{NTD}_{\text {mean }} 0.6(0.2)$ vs $0.6(0.1)(p=0.41)$, LAD $\mathrm{NTD}_{\text {mean }} 3.8(2.9)$ vs $3.5(2.3)(p=0.18), \mathrm{LAD}_{\max } 32.6(11.5)$ vs $30.6(12.4)(p=0.18)$, ipsilateral lung NTD $_{\text {mean }} 4.2(0.7)$ vs 4.2 (0.7) $(p=0.53)$, whole lung $\mathrm{NTD}_{\text {mean }} 2.0(0.3)$ vs $2.0(0.3)$ $(p=0.22)$. Fig. 1 demonstrates the heart-sparing effect of DIBH.

The number of patients in whom the median of the differences in patient comfort scores at each timepoint (CT, first and last fractions) was $<0$ ( 1 ), 0 (9) or $>0$ (12), $p=0.007$ (i.e. patients found v_DIBH more comfortable) and for radiographer satisfaction scores $<0$ (9), 0 (11) or $>0$ (3), $p=0.03$ (i.e. radiographers found v_DIBH more satisfactory).

Mean planning-CT session times (minutes) were 27 (ABC_DIBH) and $24\left(v_{-}\right.$DIBH) $(p=0.02)$. Mean treatment setup times were 11 $\left(\right.$ ABC_DIBH) and 9 ( $v_{-}$DIBH) $(p=0.04)$, mean treatment times (first beam onto last beam off) were $7 \mathrm{~min}$ for both techniques $(p=0.08)$, mean dismount time 2 min for both $(p=0.97)$. Mean total treatment time per session was $19 \mathrm{~min}$ for both techniques $(p=0.62)$.

\section{Discussion}

This randomised crossover study compared ABC_DIBH and v_DIBH in terms of setup reproducibility, normal tissue sparing and feasibility of delivery. Our results demonstrate that the two techniques are similar in terms of these parameters. Consistent with other published data, both techniques were well tolerated $[15,18]$. Baseline data (Table S1, supplementary material) suggest that target volume outlining was consistent. Lung volumes were similar for both techniques, minimising any effect on calculated cardiac doses. Heart volumes were significantly smaller with v_DIBH, suggesting a different physiological response between a machine-initiated breath-hold (ABC_DIBH) and a voluntarily-initiated breath-hold (v_DIBH).

Treatment setup errors were consistent with other studies of DIBH which have used EPI for verification $[18,29]$, but smaller than many free-breathing studies [30], suggesting that DIBH is at least as reproducible as standard free-breathing breast radiotherapy. In this study, errors were greatest in the $u$-direction, which is subject to the most change with variations in depth of DIBH or breathing pattern (e.g. chest vs abdominal breathing).

Population MD, $\Sigma$ and $\sigma$ derived from CBCT data were greater than errors derived from EPI. EPI is known to underestimate setup errors in breast cancer patients due to differences in visibility of anatomical landmarks between the two imaging techniques, time taken to acquire images and the fact that CBCT provides 3D anatomical information [31]. The inability to interrupt $\mathrm{CBCT}$ acquisition resulted in some image blurring and compromised matching. Chest wall deformation during respiration meant that chest wall matches were subject to greater errors than clip-based matches. In view of this, it is likely that CBCT-derived setup errors in this study are an overestimate of the true setup errors associated 
Table 1

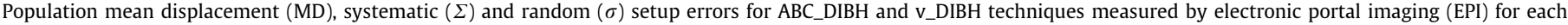
beam and in the $(u, v)$-plane $(\mathrm{mm})$.

\begin{tabular}{|c|c|c|c|c|c|c|c|}
\hline & & \multicolumn{3}{|c|}{ Right anterior oblique beam (RAO) } & \multicolumn{3}{|c|}{ Left posterior oblique beam (LPO) } \\
\hline & & ABC_DIBH & v_DIBH & $p$ & ABC_DIBH & v_DIBH & $p$ \\
\hline \multirow[t]{3}{*}{$u$-direction } & MD & -0.5 & -0.2 & 0.52 & 0.5 & 0.1 & 0.49 \\
\hline & $\Sigma$ & 1.9 & 1.8 & & 2.0 & 1.5 & \\
\hline & $\sigma$ & 2.2 & 2.1 & 0.74 & 2.4 & 2.5 & 0.24 \\
\hline \multirow[t]{3}{*}{$v$-direction } & MD & 0.7 & 0.5 & 0.63 & 0.9 & 0.8 & 0.79 \\
\hline & $\Sigma$ & 2.0 & 1.7 & & 1.9 & 1.5 & \\
\hline & $\sigma$ & 2.0 & 1.7 & 0.46 & 2.0 & 2.0 & 0.83 \\
\hline
\end{tabular}

Total number of EPIs: 665.

Table 2


ABC_DIBH and v_DIBH techniques (mm).

\begin{tabular}{|c|c|c|c|c|c|c|c|}
\hline & & \multicolumn{3}{|c|}{ Chest wall match } & \multicolumn{3}{|c|}{ Clip-based match } \\
\hline & & ABC_DIBH & v_DIBH & $p$ & ABC_DIBH & v_DIBH & $p$ \\
\hline \multirow[t]{3}{*}{ Right-left (R-L) } & MD & 0.3 & 0.5 & 0.78 & 0.4 & 0.4 & 0.93 \\
\hline & $\Sigma$ & 4.4 & 2.5 & & 3.2 & 2.4 & \\
\hline & $\sigma$ & 3.8 & 2.4 & 0.07 & 2.3 & 2.3 & 0.99 \\
\hline \multirow[t]{3}{*}{ Superior-inferior (S-I) } & MD & 2.3 & 3.4 & 0.32 & -0.1 & 1.7 & 0.10 \\
\hline & $\Sigma$ & 4.9 & 3.9 & & 2.9 & 3.6 & \\
\hline & $\sigma$ & 3.3 & 4.1 & 0.62 & 3.4 & 2.7 & 0.42 \\
\hline \multirow[t]{3}{*}{ Anterior-posterior (A-P) } & MD & -1.7 & 0.3 & 0.03 & -1.8 & 0.6 & 0.01 \\
\hline & $\Sigma$ & 3.3 & 2.8 & & 2.7 & 3.0 & \\
\hline & $\sigma$ & 2.6 & 2.7 & 0.76 & 3.5 & 2.7 & 0.53 \\
\hline
\end{tabular}

Total number of CBCTs: 126.

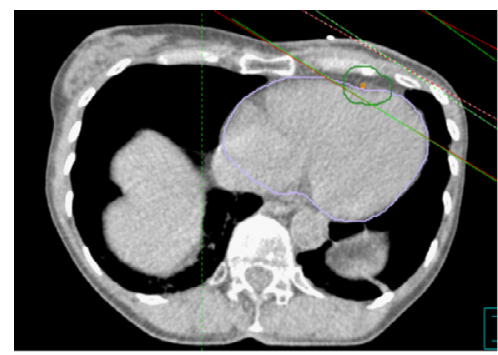

Free breathing

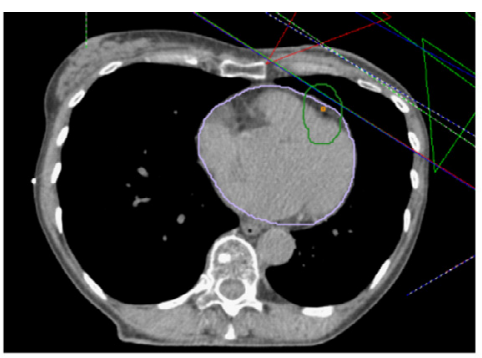

V_DIBH

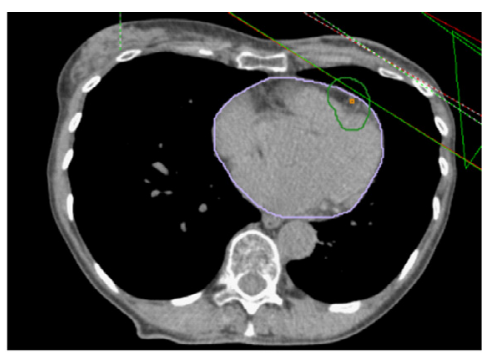

ABC_DIBH

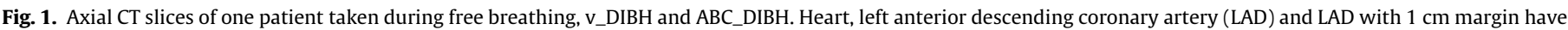
been outlined. Key: magenta - heart, orange - LAD, dark green - LAD plus $1 \mathrm{~cm}$ margin.

with these techniques. Clip-based match errors were better than DIBH setup data reported by another centre [29]; in relation to free-breathing data, $\sigma$ were similar but $\Sigma$ greater than previously reported [32]. The difference in MD in the AP direction suggests imprecision of the $\mathrm{ABC}$ equipment and/or procedure. Possible explanations for this include inaccurate spirometry or balloon valve cut-off and the inability of the $\mathrm{ABC}$ device to control breathing pattern or chest wall shape.

Our results suggest that there is no significant difference between ABC_DIBH and v_DIBH in terms of normal tissue sparing. Cardiac doses reported in this study were lower than in other published data [29,33-36], although comparison is not straightforward due to differences in target volumes and LAD margins. Ipsilateral lung doses were consistent with other published works [29,34], although whole lung doses are not widely reported.

By freeing patients of equipment, $v_{-}$DIBH was seen as more comfortable than ABC_DIBH. Patients found the mouthpiece and nose-clip used in ABC_DIBH claustrophobic. One patient with dentures found the $A B C$ mouthpiece difficult to grip. Given that
V_DIBH was a new technique to our department (unlike ABC_DIBH) it was encouraging that radiographers found v_DIBH more satisfactory than ABC_DIBH.

The timing data demonstrate small but significant advantages with $v_{-}$DIBH at planning-CT and treatment setup. The difference at CT can be accounted for by the need to set an inspiratory threshold for ABC_DIBH at this session (not required for v_DIBH), and at treatment setup by the fact that a radiographer must leave the room to operate the $A B C$ device. Treatment times for both techniques are likely to be shorter than suggested as CBCTs are not part of standard treatment.

$A B C \_D I B H$ is expensive due to capital investment in the device itself and the ongoing costs of single-use mouthpieces. The v_DIBH technique described here is a less costly alternative as no specialised equipment is required. v_DIBH currently takes longer to deliver than our centre's free-breathing technique although treatment times are expected to fall with increasing experience. This is the subject of an ongoing service evaluation within our institution. Whilst the capital costs associated with ABC_DIBH are a major 
factor in preventing its widespread uptake across the UK National Health Service (NHS), the results presented here should reassure centres with $A B C$ about the reproducibility and normal tissue sparing of ABC_DIBH in left breast radiotherapy. Otherwise, v_DIBH appears to be an effective, reproducible, comfortable and less costly alternative. The feasibility of rolling out v_DIBH to other UK centres will now be assessed in the context of the UK NCRI FAST-Forward trial (HeartSpare II), with the ultimate aim of increasing availability of heart-sparing breast radiotherapy in the UK. Further work is needed to evaluate the role of $\mathrm{v}_{-}$DIBH in breast radiotherapy for nodal irradiation and partial breast treatment.

\section{Conclusion}

Our data suggest that ABC_DIBH and v_DIBH are comparable in terms of positional reproducibility and normal tissue sparing. Patients find v_DIBH more comfortable than ABC_DIBH, and radiographers find v_DIBH more satisfactory. v_DIBH offers a time advantage at planning-CT sessions and treatment setup, and is cheaper as no specialised equipment is required.

\section{Conflict of interest}

None declared.

\section{Acknowledgements}

The authors are grateful to Dr. Liesbeth Boersma and colleagues at the Maastro Clinic for their advice on the v_DIBH technique. This article presents independent research funded by the National Institute for Health Research (NIHR) under its Research for Patient Benefit (RfPB) Programme (Grant Reference Number PB-PG-101023003). The views expressed are those of the author(s) and not necessarily those of the NHS, the NIHR or the Department of Health. The work was undertaken in The Royal Marsden NHS Foundation Trust which receives a proportion of its funding from the NHS Executive; the views expressed in this publication are those of the authors and not necessarily those of the NHS executive. We acknowledge NHS funding to the NIHR Biomedical Research Centre and the support of the NIHR, through the South London Cancer Research Network.

\section{Appendix A. Supplementary data}

Supplementary data associated with this article can be found, in the online version, at http://dx.doi.org/10.1016/j.radonc.2013. 04.021.

\section{References}

[1] Ferlay J, Shin H, Bray F, Forman D, Mathers C, Parkin D. GLOBOCAN 2008 v1.2, cancer incidence and mortality worldwide: IARC CancerBase no. 10 [Internet]. Lyon: International Agency for Research on Cancer; 2010.

[2] Maddams J, Utley M, Moller H. Projections of cancer prevalence in the United Kingdom, 2010-2040. Br J Cancer 2012;107:1195-202.

[3] Darby S, McGale P, Correa C, et al. Effect of radiotherapy after breastconserving surgery on 10-year recurrence and 15-year breast cancer death: meta-analysis of individual patient data for 10,801 women in 17 randomised trials. Lancet 2011;378:1707-16.

[4] Darby SC, McGale P, Taylor CW, Peto R. Long-term mortality from heart disease and lung cancer after radiotherapy for early breast cancer: prospective cohort study of about 300,000 women in US SEER cancer registries. Lancet Oncol 2005;6:557-65.

[5] Correa CR, Litt HI, Hwang WT, Ferrari VA, Solin LJ, Harris EE. Coronary artery findings after left-sided compared with right-sided radiation treatment for early-stage breast cancer. J Clin Oncol 2007;25:3031-7.

[6] Lind PA, Pagnanelli R, Marks LB, et al. Myocardial perfusion changes in patients irradiated for left-sided breast cancer and correlation with coronary artery distribution. Int J Radiat Oncol Biol Phys 2003;55:914-20.
[7] Nilsson G, Holmberg L, Garmo H, et al. Distribution of coronary artery stenosis after radiation for breast cancer. J Clin Oncol 2012;30:380-6.

[8] Bentzen SM, Agrawal RK, Aird EG, et al. The UK standardisation of breast radiotherapy (START) trial A of radiotherapy hypofractionation for treatment of early breast cancer: a randomised trial. Lancet Oncol 2008;9:331-41.

[9] Bentzen SM, Agrawal RK, Aird EG, et al. The UK standardisation of breast radiotherapy (START) trial $\mathrm{B}$ of radiotherapy hypofractionation for treatment of early breast cancer: a randomised trial. Lancet 2008;371:1098-107.

[10] Clarke M, Collins R, Darby S, et al. Effects of radiotherapy and of differences in the extent of surgery for early breast cancer on local recurrence and 15-year survival: an overview of the randomised trials. Lancet 2005;366:2087-106.

[11] Sixel KE, Aznar MC, Ung YC. Deep inspiration breathhold to reduce irradiated heart volume in breast cancer patients. Int J Radiat Oncol Biol Phys 2001;49:199-204.

[12] Remouchamps VM, Vicini FA, Sharpe MB, Kestin LL, Martinez AA, Wong JW. Significant reductions in heart and lung doses using deep inspiration breathhold with active breathing control and intensity-modulated radiation therapy for patients treated with locoregional breast irradiation. Int J Radiat Oncol Biol Phys 2003;55:392-406.

[13] Krauss DJ, Kestin LL, Raff G, et al. MRI-based volumetric assessment of cardiac anatomy and dose reduction via active breathing control during irradiation for left-sided breast cancer. Int J Radiat Oncol Biol Phys 2005;61:1243-50.

[14] Korreman SS, Pedersen AN, Nottrup TJ, Specht L, Nystrom H. Breathing adapted radiotherapy for breast cancer: comparison of free breathing gating with the breath-hold technique. Radiother Oncol 2005;76:311-8.

[15] Pedersen AN, Korreman S, Nyström H, Specht L. Breathing adapted radiotherapy of breast cancer: reduction of cardiac and pulmonary doses using voluntary inspiration breath-hold. Radiother Oncol 2004;72:53-60.

[16] Korreman SS, Pedersen AN, Aarup LR, Nottrup TJ, Specht L, Nystrom H. Reduction of cardiac and pulmonary complication probabilities after breathing adapted radiotherapy for breast cancer. Int J Radiat Oncol Biol Phys 2006;65:1375-80.

[17] van der Laan HP, Hurkmans CW, Kuten A, Westenberg HA. Current technological clinical practice in breast radiotherapy; results of a survey in EORTC-radiation oncology group affiliated institutions. Radiother Oncol 2010;94:280-5.

[18] Remouchamps VM, Letts N, Vicini FA, et al. Initial clinical experience with moderate deep-inspiration breath hold using an active breathing control device in the treatment of patients with left-sided breast cancer using external beam radiation therapy. Int J Radiat Oncol Biol Phys 2003;56:704-15.

[19] Nutting CM, Khoo VS, Walker V, et al. A randomised study of the use of a customised immobilisation system in the treatment of prostate cancer with conformal radiotherapy. Radiother Oncol 2000;54:1-9.

[20] Yarnold J, Coles C, On behalf of the IMPORT HIGH Trial Management Group. Intensity modulated and partial organ radiotherapy. Randomised trial testing dose escalated intensity modulated radiotherapy for women treated by breast conservation surgery and appropriate systemic therapy for early breast cancer. Planning pack, version 1. Sutton, Surrey, UK: Institute of Cancer Research; 2009. p. 1-26. Available from: http://rttrialsqa.dnsalias.org/IMPORT \%20HIGH_files/4\%5B1\%5D.2\%20Planning\%20Pack\%20IMPORT\%20HIGH\%20 final\%20version\%201.0\%2020090330.pdf.

[21] Taylor CW, Nisbet A, McGale P, Darby SC. Cardiac expo sures in breast cancer radiotherapy: 1950s-1990s. Int J Radiat Oncol Biol Phys 2007;69:1484-95.

[22] Taylor CW, Povall JM, McGale P, et al. Cardiac dose from tangential breast cancer radiotherapy in the year 2006. Int J Radiat Oncol Biol Phys 2008;72:501-7.

[23] Kirby AM, Evans PM, Donovan EM, Convery HM, Haviland JS, Yarnold JR. Prone versus supine positioning for whole and partial-breast radiotherapy: a comparison of non-target tissue dosimetry. Radiother Oncol 2010;96:178-84.

[24] International Commisssion on Radiation Units and Measurements. ICRU Report 62. Prescribing, recording and reporting photon beam therapy (Supplement to ICRU Report 50). Bethesda, MD: ICRU, 1999.

[25] Scrimger RA, Tome WA, Olivera GH, Reckwerdt PJ, Mehta MP, Fowler JF. Reduction in radiation dose to lung and other normal tissues using helical tomotherapy to treat lung cancer, in comparison to conventional field arrangements. Am J Clin Oncol 2003;26:70-8.

[26] Donovan EM, Castellano I, Eagle S, Harris E. Clinical implementation of kilovoltage cone beam $\mathrm{CT}$ for the verification of sequential and integrated photon boost treatments for breast cancer patients. $\mathrm{Br} \mathrm{J}$ Radiol 2012;85:e1051-1057.

[27] Penninkhof J, Quint S, Baaijens M, Heijmen B, Dirkx M. Practical use of the extended no action level (eNAL) correction protocol for breast cancer patients with implanted surgical clips. Int J Radiat Oncol Biol Phys 2012;82:1031-7.

[28] van Herk M. Errors and margins in radiotherapy. Semin Radiat Oncol 2004;14:52-64.

[29] Borst GR, Sonke JJ, den Hollander S, et al. Clinical results of image-guided deep inspiration breath hold breast irradiation. Int J Radiat Oncol Biol Phys 2010;78:1345-51.

[30] Hurkmans CW, Remeijer P, Lebesque JV, Mijnheer BJ. Set-up verification using portal imaging; review of current clinical practice. Radiother Oncol 2001;58:105-20.

[31] Topolnjak R, Sonke JJ, Nijkamp J, et al. Breast patient setup error assessment: comparison of electronic portal image devices and cone-beam computed tomography matching results. Int J Radiat Oncol Biol Phys 2010;78: $1235-43$. 
[32] Kirby AM, Evans PM, Helyer SJ, Donovan EM, Convery HM, Yarnold JR. A randomised trial of Supine versus Prone breast radiotherapy (SuPr study): comparing set-up errors and respiratory motion. Radiother Oncol 2011;100:221-6.

[33] Hayden AJ, Rains M, Tiver K. Deep inspiration breath hold technique reduces heart dose from radiotherapy for left-sided breast cancer. J Med Imaging Radiat Oncol 2012;56:464-72.

[34] Vikstrom J, Hjelstuen MH, Mjaaland I, Dybvik KI. Cardiac and pulmonary dose reduction for tangentially irradiated breast cancer, utilizing deep inspiration breath-hold with audio-visual guidance, without compromising target coverage. Acta Oncol 2011;50:42-50.
[35] Hjelstuen MH, Mjaaland I, Vikstrom J, Dybvik KI. Radiation during deep inspiration allows loco-regional treatment of left breast and axillarysupraclavicular- and internal mammary lymph nodes without compromising target coverage or dose restrictions to organs at risk. Acta Oncol 2012;51:333-44.

[36] Johansen S, Vikstrom J, Hjelstuen MH, Mjaaland I, Dybvik KI, Olsen DR. Dose evaluation and risk estimation for secondary cancer in contralateral breast and a study of correlation between thorax shape and dose to organs at risk following tangentially breast irradiation during deep inspiration breath-hold and free breathing. Acta Oncol 2011;50:563-8. 